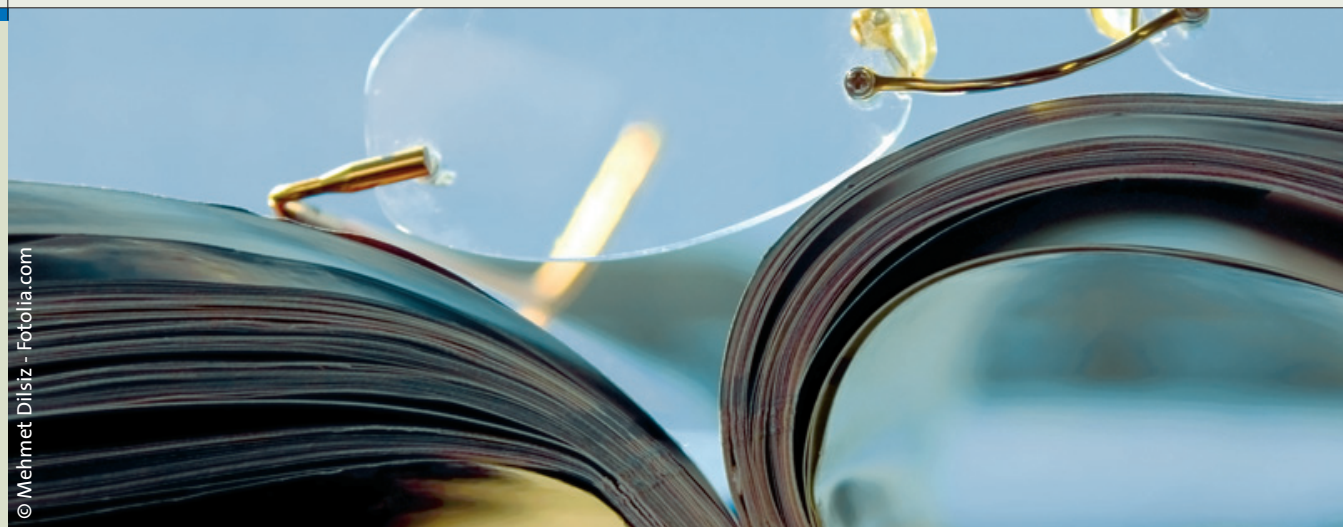

\section{Pilotversuch mit Azathioprin beim kreisrunden Haarausfall}

Bei der Alopecia areata (AA) spielen T-Zell-vermittelte Immunreaktionen eine Rolle, die gegen ein (unbekanntes) Antigen des Haarfollikels gerichtet sind. Daher könnte theoretisch das Immunsuppressivum Azathioprin eine Therapieoption sein, dem (neben seiner antiproliferativen Wirkung auf BZellen) ein spezieller gegen T-Lymphozyten gerichteter Effekt zugeschrieben wird.

A nekdotisch hat Azathioprin bei Morbus-Crohn-Patienten, die gleichzeitig eine $\mathrm{AA}$ aufwiesen, auch neues Haarwachstum induziert. Dies war u.a. der Grund, warum Hautärzte der Universität Teheran den Ansatz prüften.

20 Patienten im Alter von 8 bis 57 Jahren wurden in die offene Pilotstudie aufgenommen, die Alopecia areata mit kahlen Stellen von mindestens 20\% des behaarten Kopfes aufwiesen. Die gegenwärtige AA-Episode dauerte bei den Teilnehmern bereits zwölf bis 84 Monate; bei jedem zweiten war anamnestisch eine über zwei Jahre dauernde Alopecia areata (oder totalis oder universalis) bekannt.

Studienmedikation war $2 \mathrm{mg}$ des Antimetaboliten pro kg Körpergewicht. Damit erzielte man nach sechs Monaten eine mittlere Rate an neuem Haarwachstum von $52,3 \%$. Der Haarverlust vor Therapiebeginn betrug gemittelt über die ganze Kohorte 72,7\%; nach sechs Monaten Azathioprin-Behandlung waren es $33,5 \%$ - ein statistisch hochsignifikanter Effekt. Gemäß SALT, dem Severity of Alopecia Tool, war der mittlere Haarverlust-Score initial 3,9, am Ende der Therapiephase 1,8 ( 0 bedeutet kein Haarverlust, 5 vollständigen Haarverlust).

Beide Geschlechter hatten ähnliche Resultate. Tendenziell schienen weniger schwere Fälle mit kürzerer Krankheitsdauer besser anzusprechen. Eine sichtbare Wirkung stellte sich meist nach drei Monaten ein.

Eine Patientin musste die Therapie wegen Verschlechterung der Leberfunktion vorzeitig absetzen. Bei drei weiteren Teilnehmern reduzierte man die Dosis, nachdem eine milde Leukopenie festgestellt wurde. In einem Fall wurde die Dosierung wegen gastroin-
O b man nun keimarm oder als Bakterienschleuder agiert, hängt nicht nur davon ab, wie und womit man sich die Hände wäscht. Nicht minder wichtig ist, wie man sich die Hände nach dem Waschen trocknet.

Schlecht sind z.B. elektrische Handtrockner, bei denen man die Hände zum Trocknen in einem warmen Luftstrahl reibt. Oft werden die Hände dabei nicht richtig trocken. Der verbleibende Feuchtigkeitsfilm auf der Haut sorgt dafür, dass man seine Bakterien besonders effektiv verbreitet. testinaler Beschwerden verringert. Ansonsten wurde die Medikation gut toleriert. Sie wurde nach Studienende für weitere sechs Monate fortgesetzt.

Fazit: Auch wenn (reversibler) Haarausfall zu den seltenen Nebenwirkungen unter Azathioprin zählt, halten die Autoren das Purinanalogon für eine interessante Option und sprechen sich für größere Untersuchungen im Rahmen randomisierter kontrollierter Studien aus.

wpa

Farshi S et al. Could azathioprine be considered as a therapeutic alternative in the treatment of alopecia areata? A pilot study. Int J Dermatol 2010; 49: 1188-93.

\title{
Infektionsprophylaxe: Wie man sich die Hände richtig trocknet
}

\section{Gründliches Händewaschen soll die Verbreitung von Krankheitserregern eindämmen. Werden hier Fehler gemacht, erzielt man u. U. das Gegenteil des gewünschten Effektes: Man verteilt die Keime großflächig.}

Wer sich die noch feuchten Hände dann an der eigenen Kleidung abwischt, ist als Bakterienschleuder fast unschlagbar.

Mit Papierhandtüchern oder ultraschnellen elektrischen Handtrocknern, bei denen man die Hände nicht reiben muss, lässt sich die Verbreitung von Keimen hingegen am wirkungsvollsten verhindern.

de

J Applied Microbiol; DOI: 10.1111/j.13652672.2010.04838.x 